\title{
Pieris ProteoLab
}

\author{
Improving on antibodies
}

a

herapeutic antibodies have proven both difficult and expensive to manufacture. Because of the complex structure of immunoglobulins (four polypeptide chains encoded by two genes), expression on a commercial scale has been limited to mammalian cells, such as Chinese hamster ovary ( $\mathrm{CHO}$ ) cells-a costly proposition. Lipocalins are a family of storage and transport proteins with binding properties similar to antibodies. German startup Pieris hopes they could prove a cheaper alternative to antibodies.

The structure of lipocalins was first elucidated during the early 1980s: they have a stable and compact core structure (called a "beta barrel"), which is common to all lipocalins, and four variable loops, which create a binding site for a specific ligand. Because lipocalins are monomeric polypeptides and do not require additional factors for correct folding and molecular assembly, they can be produced in nonmammalian cells, such as yeast and Escherichia coli, which are tenfold less expensive to grow than mammalian cells.

In the mid-1990s, Arne Skerra, cofounder and current chairman of the board at Pieris, began modifying the variable loops of lipocal ins to produce libraries of molecules in Escherichia coli. According to Skerra, these recombinant proteins functioned rather like a cross between an antibody and a lipocalin, and so they were called anticalins. He later succeeded in creating, from a native lipocalin of the Pieris brassicae butterfly, an antical in that could bind specifically to the dye fluorescein with an antibody-like affinity (Proc. Natl. Acad.

Founded: January 2001

Founders: Arne Skerra (Chairman), Steffen Schlehuber, Claus Schalper, and Carsten Schuerrle

CEO: Martin Poehlchen

Employees: 10

Financing to date: Amount not disclosed:

TransConnect (Munich), BioM AG

(Martinsreid, Germany), tbg Deutsche

Ausgleichsbank (Bonn), and

Bayern-Kapital (Landshut, Germany)

Location: Freising-Weihenstephan,

Germany

http://www.pieris-ag.de/
Sci. USA, 96, 1898-1903, 1999). Libraries of anticalins specifically directed against protein targets have subsequently been constructed.

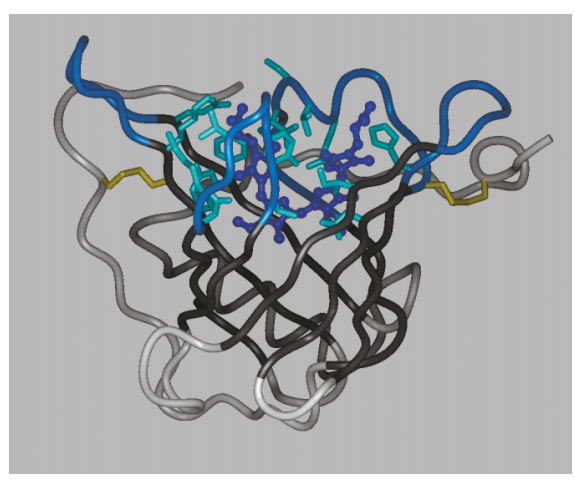

Tertiary structure of a bilin-binding anticalin with randomized amino acids depicted.

Skerra says that anticalins are potentially superior to antibodies as therapeutics because of their small size and greater stability. Antibodies are large, complicated structures, and their antigen-binding associations are often unstable; anticalins, by contrast, are compact and stable, which makes them more suitable than antibodies for use as medications. Anticalins are also small in size, enabling them to fit between cells (e.g., tightly packed cancer cells), and thus they can penetrate diseased tissues more readily than antibodies. M oreover, by fusing two anticalins to form a "duocalin," agents can be created that bind to two targets; for example, a duocalin could be used to help bind a cytotoxic lymphocyte to a tumor cell, helping to destroy a cancer.

Pieris was founded on the library of engineered anticalins begun by Skerra, which is well protected by broad patents and now numbers $\sim 10$ billion structures, each a potential therapeutic. Indeed, Pieris bucks the current trend of genomics firms that have too many targets; Skerra says Pieris is "evaluating its first anticalins against therapeutic targets in preclinical experiments but is eager to in-license further validated targets for cognate anticalin selection."

In January, Pieris struck up its first research collaboration to develop anticalins against atherosclerosis targets from CytoTools (Darmstadt, Germany). Skerra anticipates that the company will initiate more collaborations in the near future not only with therapeutics companies but also with diagnostics and, possibly, environmental science companies. U Itimately, Skerra hopes that Pieris will "graduate" into drug development; the company is currently searching for validated targets to interrogate with its anticalin library. $A B$

\section{Caprion Pharmaceuticals}

\section{Putting proteins in context}

early every therapeutic targets one of 500 human proteins, but this is a small fraction of the total number of proteins thought to be present in the human proteome (estimated at between 100,000 and $1,000,000$ ) . Discovering novel targets and their functions, however, is a tricky business. Caprion Pharmaceuticals maintains that the function of a protein is dependent on its location within a cell; proteins behave differently when in the presence of, for example, nuclear rather than cytosolic proteins. Caprion believes that its unique organelleisolation skills keep proteins in context, enabling the determination of protein function and thus easing the discovery of new drug targets.
Most proteomics companies use wholecell analyses to isolate proteins that may be associated with disease. H owever, Clarissa Desjardins, Caprion's vice president of business development, explains that the wholecell approach has several technical problems. For example, some proteins are expressed at a 10,000-fold greater concentration than others in cells; if a potential target protein is weakly expressed, then common isolation techniques (such as two-dimensional gels and mass spectrometry) will fail to detect it. In addition, a protein may not contribute to a disease unless it is in the presence of another protein; whole-cell proteomic analyses muddle data by bringing together proteins that naturally reside in different organelles and would thereforenever actually interact 